\title{
Strong Soft X-ray Emission and High Accretion Rates in Seyfert Galaxies
}

\author{
Ewa Szuszkiewicz \\ Astronomy Group, Department of Physics and Astronomy, University of \\ Leicester, University Road, Leicester, LE1 $7 R H$, England
}

\begin{abstract}
Slim accretion disk models with high accretion rates provide a natural explanation for the strong soft $\mathrm{X}$-ray emission observed in several narrow-line Seyfert 1 galaxies.
\end{abstract}

\section{Theoretical predictions of slim accretion disk models}

Fitting spectra of active galactic nuclei (AGN) with the standard, optically thick and geometrically thin accretion disk models has often led to luminosities which contradict the basic assumptions adopted in the standard model. This fact has been used as an argument against the existence of accretion disks in AGN. However, there is no obvious reason why the accretion rates in AGN should not exceed the thin disk limit. It was shown some time ago that more general ("slim") accretion disk models are self-consistent even for moderately superEddington luminosities. Using slim disk models, Szuszkiewicz, Malkan, and Abramowicz (1996) have reexamined the hypothesis that the optical/UV/soft $\mathrm{X}$-ray continuum of Active Galactic Nuclei can be interpreted as a thermal emission from an accretion disk. They find that, unlike those of low accretion rate disks, the spectra of the high accretion rate disks extend into the soft X-ray band. A comparison with observations shows that such disks could produce the soft X-ray excesses claimed in some AGN. The recent discovery of a large class of Seyfert galaxies with unusually strong $\mathrm{X}$-ray excesses, identified with narrowline Seyfert 1 galaxies, offers new possibilities for testing theoretical predictions of the models.

\section{Observational properties of narrow-line Seyfert 1 galaxies}

The ROSAT PSPC has currently identified over 30 Seyfert-type galaxies with unusually strong soft X-ray emission, among them RE J1034+396 (Puchnarewicz et al., 1995), RE J1237+264 (Brandt, Pounds, and Fink, 1995), RE $1442+352$ (Gondhalekar et al., 1994). These objects have steeply rising ROSAT spectra. They show rapid variability, strong Fe II emission and narrow permitted Balmer lines. Pounds and Brandt (1996) suggested that the underlying and common feature of the steep spectrum Seyfert class is an unusually high accretion rate.

Another interesting property of narrow-line Seyfert 1 galaxies is the similarity of their spectra to the high-state spectra of the Galactic black hole candidates 
(GBHC). This result has been found for RE 1034+396 from ROSAT and ASCA observations by Pounds, Done and Osborne (1995). If confirmed, this suggests that a very similar physical mechanism may be responsible for the observed spectra in these objects.

\section{Discussion}

The increasing number of Seyfert 1 galaxies with strong soft $\mathrm{X}$-ray emission presents a particularly powerful diagnostic of AGN and a great challenge to accretion models.

Spectra calculated from slim disk models with high accretion rates do have strong X-ray excesses. Szuszkiewicz, Malkan and Abramowicz (1996) have examined three particularly well observed, bright, low red shift quasars with a high soft X-ray flux relative to their optical emission (Mkn 509, Mkn 841 and PG $1211+143)$. Acceptable agreement between the calculated and observed spectra is found. Other objects from their sample have less pronounced soft X-ray excesses, while others show no excess at all. The relatively small number of known sources with well-observed strong soft X-ray excesses, and the controversy about the interpretation of the shape of this excess, produce a rather limited applicability for the high accretion rate models. The new class of objects identified by ROSAT, provides a long-awaited opportunity for placing meaningful constraints on such models.

Similarities in the observed appearance of the different types of accretionpowered objects are extremely important for studying the nature of the compact energy source in AGN and the matter in its immediate environment. Abramowicz, Szuszkiewicz and Wallinder (1989) have investigated the similarities in variability patterns of Galactic X-ray sources and active galactic nuclei. These are quite evident when the characteristic time scales are scaled by a factor corresponding to the mass difference. The suggestion that RE J $1034+396$ represents the Seyfert analogue to the high state of a GBHC seems to confirm the conjecture of similar basic physics in the accretion processes in Galactic black hole candidates and AGN.

In conclusion, we propose a full quantitative comparison between slim disk predictions and observed spectra of narrow-line Seyfert 1 galaxies.

\section{References}

Abramowicz, M. A., Szuszkiewicz, E., and Wallinder, F., 1989, in Theory of Accretion Disks, eds. F. Meyer et al., Kluwer Academic Publisher: Dodrecht, p.141

Brandt, W. N., Pounds, K. A., and Fink, H. H., 1995, MNRAS, 273, L47

Gondhalekar, P. et al. 1994, MNRAS, 268, 973

Pounds, K.A., and Brandt, W. N., 1996, Proc. Asca Symposium: Tokyo

Pounds, K.A., Done, C., and Osborne, J. P., 1995, MNRAS, 277, L5

Puchnarewicz et al., 1995, MNRAS, 276, 20

Szuszkiewicz, E., Malkan, M. A., and Abramowicz, M. A. 1996, ApJ, 458, 474 